\section{BLEEDING MECKEL'S DIVERTICULUM}

\section{A REPORT OF TWO CASES}

BY

JOHN N. WALTON, M.B., M.R.C.P. Late Medical Registrar

AND

NORMA D. LILL, M.B., B.S. Late Senior House Officer

The Royal Victoria Infirmary, Newcastle-upon-Tyne

A Meckel's diverticulum remains as a vestigial remnant of the vitelline duct in between 1 and $2 \%$ of individuals; Harkins (1933) found the anomaly in some form in $1.3 \%$ of over 25,000 necropsies.

Intestinal obstruction is said to be the most common complication (Taylor, 1947 ; Haber, 1947), but out of 1,605 cases from the literature Moses (1947) found that the presenting disorder was haemorrhage in $496(30.9 \%)$, intestinal obstruction in $383(23.8 \%)$, perforation in 222 (1.3.8\%), a Littré's hernia in $188(11.7 \%)$, and diverticulitis in $165(10.3 \%)$. The incidence of the whole group of disorders is more than twice as great in males as in females.

In the cases here reported the bleeding was intractable and required urgent surgical intervention.

\section{Case 1}

A salesman aged 27 was admitted to the Royal Victoria Infirmary under Dr. A. G. Ogilvie on the morning of August 23, 1950. He had woken feeling nauseated, weak, and faint, and perspiring profusely. On going to the w.c. he passed a large amount of dark semi-fluid mate:ial per rectum.

Five years previously, while in the Army in India, he had had a similar experience and had received a blood transfusion. Investigation revealed no gastro-intestinal abnormality. For the past two years he had had vague lower abdominal discomfort after meals.

On examination he looked healthy, not evidently in pain. The pulse rate was 64 a minute, blood pressure $120 / 70$. Abdominal palpation revealed nothing significant, but on rectal examination the examining finger was stained with dark-red blood. No other physical abnormality was noted.

The haemoglobin on admission was $75 \%$ (11.25 g./ $100 \mathrm{ml}$. ), Hess's capillary fragility test was negative, and the bleeding and clotting times were normal.

Some four hours after admission the patient suddenly became pale, anxious, and sweating; the pulse rate rose to 96 , and the blood pressure fell to $100 / 50$. Ten minutes later he passed much dark-red blood per rectum. After a transfusion of 2 pints (1.14 litres) of compatible whole blood he improved. It was thought that the bleeding was from a duodenal ulcer, an intestinal polyp, or a Meckel's diverticulum.

The following day he again had a severe haemorrhage, necessitating further transfusion. Professor F. H. Bentley was consulted, and agreed that if there was evidence of further bleeding an emergency laparotomy should be performed. Radiographic examination, gastroscopy, and sigmoidoscopy were, for obvious reasons, not carried out.

On the morning of August 27 the patient again had a severe haemorrhage; his haemoglobin fell to $56 \%(8.4 \mathrm{~g} . /$ $100 \mathrm{ml}$.), and another continuous drip transfusion was begun

Operation.-At 3 p.m. Professor Bentley opened the abdomen through a right para-umbilical paramedian incision; the posterior wall of the duodenum was felt to be a little thickened, but no ulcer appeared to be present. The caecum and lower ileum were filled with blood. Some $2 \mathrm{ft} .(61 \mathrm{~cm}$.) above the ileo-caecal valve a Meckel's diverticulum, about 4 in. $(10 \mathrm{~cm}$.) in length, was found protruding from the antimesenteric border of the gut. Blood filled the diverticulum and the ileum for some 12 in. $(30.5 \mathrm{~cm}$.) proximal to it ; above this point the bowel was collapsed. Since it was evident that the diverticulum was the site of the haemorrhage it was excised.

The mucosa of the last inch $(2.5 \mathrm{~cm}$.) of the diverticulum showed multiple petechial haemorrhages and two superficial ulcers each about $3 \mathrm{~mm}$. in diameter; in the base of one of these could be seen the mouth of a small vessel. The proximal portion was macroscopically normal. Dr. A. $\mathbf{H}$. Cameron reported that histological examination showed acute ulceration, with no evidence of gastric heterotopia.

The patient made a good recovery, although gastric aspiration and intravenous therapy were necessary for 36 hours owing to moderate abdominal distension. The stools were free from occult blood within five days. On discharge on September 11 he felt well and his haemoglobin was $96 \%$ (14.4 g./100 ml.).

\section{Case 2}

A male telephonist aged 17 was admitted to the Royal Victoria Infirmary at 2.30 a.m. on July 15,1951 , under the care of Mr. John Brumwell. He had been perfectly well until two days before admission, when he began to pass small quantities of dark-red blood per rectum. This was unaccompanied by other symptoms until 24 hours later, when he began to feel weak and faint and to sweat profusely. At 1.45 o'clock on the morning of admission he woke with an urgent desire to move his bowels, fainted on getting out of bed, and passed a further large quantity of dark-red blood.

On admission he was anxious, sighing, and restless but not in pain. His conjunctivae and mucous membranes were blanched, pulse rate 120 , and blood pressure $100 / 60$. There was no tenderness or mass on abdominal palpation, but on rectal examination the examining finger was stained with dark-red blood.

A blood transfusion was begun immediately, and over the next 24 hours he received 9 pints $(5.1$ litres) of whole blood, with considerable improvement. At one stage his haemoglobin had fallen to $45 \%(6.5 \mathrm{~g} . / 100 \mathrm{ml}$.).

On July 20 he was transferred to a medical ward for investigation under the care of Dr. A. G. Ogilvie. His general condition was then good; pulse 85 , blood pressure $130 / 70$, and haemoglobin $66 \%$ (9.5 g./100 ml.).

Two days later he suddenly became weak and faint, vomited clear watery material, and passed a large darkred fluid stool; despite continuous transfusion the bleeding continued. The clinical picture was so reminiscent of Case 1 that a firm clinical diagnosis of bleeding from a Meckel's diverticulum was made. Mr. Denis Hindmarsh was consulted, and agreed to carry out laparotomy.

Operation.-The abdomen was opened through a right paramedian incision. The large intestine and lower ileum were found to be filled with blood. A Meckel's diverticulum was identified, about 2 in. $(5 \mathrm{~cm}$.) in length, and the distal end was clearly thickened; it was removed together with a "wedge" of intestinal wall.

Dr. J. Hart Mercer reported on the specimen as follows: "The diverticulum shows a fairly deep oval ulcer in the floor of which no obvious vessel is visible to the naked eye. The mucosa macroscopically is mostly of the small intestinal type, but at the edge of the specimen some distance from the ulcer a thicker mucosa of more luxuriant appearance is seen. Histological examination reveals an active chronic peptic ulcer: the mucosa immediately surrounding it is of intestinal type, but further away gastric mucosa is seen." 
The patient made a good and uneventful recovery from the operation, and on discharge on August 3 his haemoglobin was $89 \%$ (12.9 g./100 ml.).

\section{Discussion}

In the reported cases of haemorrhage from a Meckel's diverticulum a correct pre-operative diagnosis has rarely been made, except in children. In Case 1, although ulceration in a diverticulum was considered as a possibility, no firm diagnosis was reached before operation ; in the second case, however, the similarity of the clinical picture to that in the first patient was striking and enabled the diagnosis to be made confidently. The character of the blood passed per rectum weighed heavily in pointing out the offending organ ; it.was dark red in colour, intermediate between the characteristic tarry stool of melaena from a duodenal ulcer and the bright-red blood of haemorrhage from a lesion of the colon. This point has been stressed by Murray (1947). A second feature of value in the differential diagnosis was that both patients vomited clear watery fluid containing no blood.

Schaetz (1925) described the presence of areas of heterotopic gastric mucosa in 5 out of 30 diverticula examined, and it has been assumed that, as occurred in our Case 2, haemorrhage is invariably the result of ulceration in or around such islands (Haber, 1947 ; Taylor, 1947). However, Chesterman (193-5) pointed out that ulceration can occur in the absence of gastric mucosa, explainied by Winkelbauer (1929) as being due to irregular peristalsis producing invagination of the apex of the diverticulum ; this is possibly the explanation in Case 1, as the lesions were confined to the tip.

Haemorrhage from a Meckel's diverticulum may produce a serious threat to life. It should be suspected in patients suffering from melaena of the type described ; if haemorrhage is recurrent laparotomy is indicated, since the risks of an adequately performed operation are slight. If surgical treatment is not required in the acute stage a Meckel's diverticulum may occasionally be demonstrated radiologically (Rousseau and Martin, 1943).

\section{Summary}

Two cases of bleeding due to ulceration in a Meckel's diverticulum are reported. Cure followed excision of the diverticulum in each case.

One specimen showed acute ulceration and no gastric heterotopia; the other contained gastric mucosa, and a chronic peptic ulcer was present.

It is suggested that the character and colour of the blood passed per rectum-that is, dark red, as distinct from the tarry stool of a duodenal ulcer and the bright-red blood of a colonic lesion-may enable this syndrome to be diagnosed pre-operatively.

We are indebted to Dr. A. G. Ogilvie for permission to publish these cases, to Professor F. H. Bentley and Mr. Denis Hindmarsh for details of the surgical treatment, and to Dr. A. H. Cameron and Dr. J. Hart Mercer for the pathological reports.

\section{REFERENCES}

Chesterman, J. T. (1935). Brit. J. Surg., 23. 267.

Haber, J. J. (1947). Amer. J. Surg., 73, 468.

Harkins, H. N. (1933). Ann. Surg., 98, 1070.

Moses, W. R. (1947). New Engl. J. Med., 237, 118.

Murray, A. G. (1947). Med. J. Aust., 1, 145.

Rousseau, J. P., and Martin, A. G. M. (1943). Radiology, 40, 605.

Schaetz, G. (1925). Quoted by Chesterman (1935).

Taylor, S. (1947). Lancet, 2, 786.

Winkelbauer (1929). Quoted by Chesterman (1935).

\section{Medical Memoranda}

\section{Accelerated Ossification in a Cretin caused by High Thyroid Dosage}

The following case is reported because it is not generally known, and is not cited in paediatric textbooks, that high thyroid dosage in cretinism may cause advanced ossification, and because it demonstrates the advisability, during treatment, of taking serial radiographs of the ossific centres if maximal dosage within the limits of clinical tolerance is to be given.

\section{Case Report}

The patient was a full-term female infant with a birth weight of $6 \mathrm{lb} .13 \mathrm{oz}$. $(3.1 \mathrm{~kg}$.). She came under observation at 14 weeks of age, when the mother reported that the baby had seemed normal until 10 to 12 .weeks, when she became drowsy, stopped smiling, lost interest, developed a feeble hoarse cry, and altered in facial appearance. These symptoms had since progressed, and on examination the baby, which then weighed $8 \mathrm{lb} .7 \mathrm{oz}$. $(3.8 \mathrm{~kg}$.), was seen to be a typical cretin (Figs. 1 and 2), with lethargy, coarse expressionless features, thick protruding tongue, dry yellow skin, puffy hands, large abdomen, and umbilical hernia. She did not watch other persons, the eyes did not follow a moving object, and there was no smile. In the prone position the head slumped down, and in no position of the body was it held steady. Radiographs showed gross delay in ossification in that no centres were visible for the cuboids, the heads of the tibiae, or the wrists.

It was decided to increase thyroid dosage as quickly as practicable to the limits of clinical tolerance on the grounds $(a)$ that often the mental development of cretins does not progress favourably on the customary schedules even when treatment begins early (Warkany, 1950); (b) that some poor results may be due to underdosage attributable to faulty assay, deterioration, or impaired absorption ; and $(c)$ that other poor results may be due to initial slowness in building the dose to a maintenance level.

The initial treatment took place in hospital, dry thyroid (B.P.) being given in doses of $1 ; 10, \frac{1}{8}, \frac{1}{4}$, and $\frac{1}{2} \mathrm{gr}$.
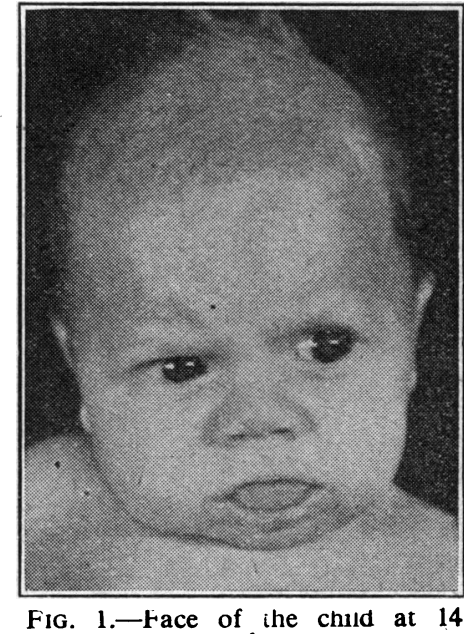
weeks.

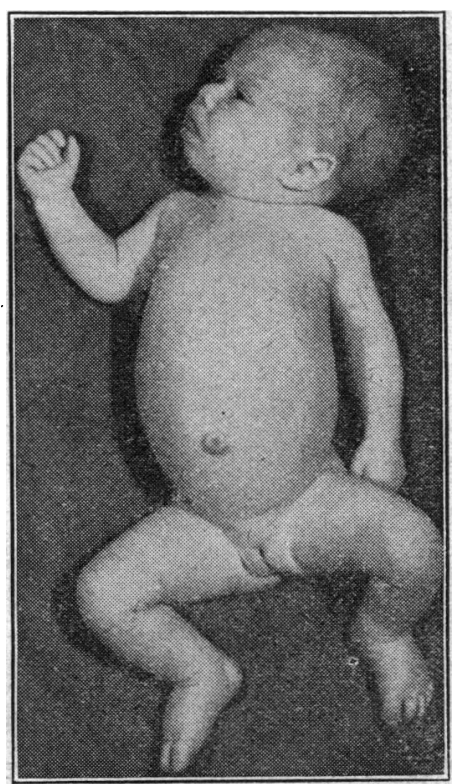

FIG. 2.- I he child al it weeks. 